\title{
Feeling socially powerless makes you more prone to bumping into things on the right and induces leftward line bisection error
}

\author{
DaVID WiLkinson \\ University of Kent, Canterbury, England \\ Ana Guinote \\ University College London, London, England \\ AND \\ Mario Weick, Rosanna Molinari, and Kylee Graham \\ University of Kent, Canterbury, England
}

\begin{abstract}
Social power affects the manner in which people view themselves and act toward others, a finding that has attracted broad interest from the social and political sciences. However, there has been little interest from those within cognitive neuroscience. Here, we demonstrate that the effects of power extend beyond social interaction and invoke elementary spatial biases in behavior consistent with preferential hemispheric activation. In particular, participants who felt relatively powerless, compared with those who felt more powerful, were more likely to bisect horizontal lines to the left of center, and bump into the right-hand (as opposed to the left-hand) side when walking through a narrow passage. These results suggest that power induces hemispheric differences in visuomotor behavior, indicating that this ubiquitous phenomenon affects not only how we interact with one another, but also how we interact with the physical world.
\end{abstract}

Power has been defined as the ability to influence others (Lewin, 1941) or control others' outcomes by providing or withholding resources, be these material (e.g., food/shelter) or social (e.g., knowledge/affection) in nature (Fiske, 1993; Keltner, Gruenfeld, \& Anderson, 2003; Thibaut \& Kelley, 1959). Social psychological research has shown that power affects the propensity to act in social situations. Less powerful people are slower than their more powerful counterparts to determine the appropriate course of action; slower to initiate goal-directed activity; and more distracted by irrelevant cues during goal pursuit (Guinote, 2007a, 2008). These qualities are thought to arise from a greater dependency on others, which channels working memory resources away from preparing and implementing one's own actions toward monitoring those of others (Guinote, 2007a). The increased perception of threat experienced by less powerful people may also inhibit action (Galinsky, Gruenfeld, \& Magee, 2003; Keltner et al., 2003).

Intriguingly, recent data suggest that power may not only affect the willingness and motivation to act. Rather, it may also affect action in a more fundamental way, having an impact on basic sensorimotor processes involved in movement control. This suggestion is based on the finding that changes in social power alter the distribution of activ- ity across the two sides of the brain, so that heightened power preferentially activates the left hemisphere and reduced power preferentially activates the right hemisphere (Boksem, Smolders, \& De Cremer, in press). This physiological observation is important because we know that asymmetries in hemispheric activation can directly affect how physical actions are carried out. One notable effect is a shift in behavior toward the side of space opposite the activated hemisphere (Kinsbourne, 1993). In clinical patients for whom the asymmetry arises from a unilateral brain lesion, this "spatial neglect" can lead to near total failure to act toward stimuli on one side, causing problems in eating, dressing, and navigation (Wilson, Cockburn, \& Halligan, 1987). In healthy volunteers, the "pseudoneglect" induced by unimanual movement also influences, albeit to a lesser degree, which physical stimuli are selected as targets for action (Nicholls, Loftus, Mayer, \& Mattingley, 2007).

In the present context, it is important to establish whether power induces pseudoneglect, not only because it would lend initial support to the argument that the behavioral effects of power can be partly predicted by the underlying pattern of hemispheric specialization, but also because it would raise the possibility that power has an impact on the performance of simple sensorimotor tasks,

D.Wilkinson, dtw@kent.ac.uk 
such as reaching and walking, that are ubiquitous in daily routine and goal pursuit. To date, the effects of power on cognitive processing have been restricted to tasks involving perceptual discrimination, memory, and problem solving that did not measure lateral spatial bias, and mostly required simple buttonpress responses (e.g., Guinote, 2007b; Guinote, Reese, \& Wilkinson, in press; Smith, Jostmann, Galinsky, \& van Dijk, 2008).

The idea that social power modulates hemispheric activity stems from the motivational account of Keltner et al. (2003), who proposed that high power facilitates approachrelated behaviors that are sensitive to reward and positive affect, whereas low power facilitates inhibitory, withdrawing behaviors related to threat and punishment. Biochemical studies suggest that approach-related behaviors elicited by powerful feelings are associated with increased dopamine activity in the left hemisphere, whereas the inhibition-related behaviors elicited by powerlessness are associated with increased norepinephrine activity in the right hemisphere (Ashby, Isen, \& Turken, 1999; Davidson, 1992). Recent EEG data appear to support this distinction, demonstrating that individuals primed to feel powerful show stronger left frontal activity than do those primed to feel powerless (Boksem et al., in press). The key, unanswered questions are whether these differences in hemispheric activation are sufficient to affect outward physical behavior, and if so, how? There are many instances from neuroimaging in which a change in hemispheric activation is not associated with a change in observable behavior, a finding taken to suggest that not all brain activity is functionally relevant (see Wilkinson \& Halligan, 2004). Thus, even though high and low power have been shown to preferentially activate different cerebral hemispheres, it is not known whether either form of activation is sufficient in magnitude or type to affect overt behavior.

In the present study, we therefore conducted two experiments to determine whether social power induces a lateralized spatial bias in manual activity. In the first experiment, we employed a sensitive, conventional measure of left/ right bias, the line bisection task (Robertson \& Halligan, 1999). Here, a tendency to mark toward one end of the line is taken to reflect the dominance of the contralateral hemisphere, whereby leftward deviation reflects stronger right-hemisphere control, and rightward deviation reflects stronger left-hemisphere control (Kinsbourne, 1993). In the second experiment, we looked for corresponding changes in a task requiring gross locomotory activity that might be regarded as more functionally relevant. Here, participants were instructed to walk through a narrow passage while balancing a full cup of water on a small tray. A distractor task, such as cup balancing, is commonly used in studies of this nature to distract participants from the primary measure of left/right bumping and thus more accurately capture underlying spatial asymmetries (see Nicholls et al., 2007; Nicholls, Loftus, Orr, \& Barre, 2008). As with manual line bisection, stronger activation of the right hemisphere should push attention leftward, which may lead to a neglect of right space and increased likelihood of bumping into objects on that side.
As mentioned above, it was unclear whether high or low power (or both) would elicit a lateralized spatial bias. Although some studies have shown stronger behavioral effects in individuals primed with high power (e.g., Galinsky et al., 2003; Guinote, 2007b; Keltner et al., 2003), others have reported stronger effects in participants primed with low power (e.g., Guinote et al., in press; Smith et al., 2008). As a consequence, our hypothesis was relatively generic in nature; given that high and low power are taken to activate the left and right hemispheres, respectively (Boksem et al., in press; Keltner et al., 2003), we predicted that any effect of low power would be to shift attention left, whereas any effect of high power would be to shift it right.

\section{METHOD}

\section{Participants}

Forty-one female and 31 male university students, mean age 22 years, were recruited for the line bisection study (Experiment 1), and a separate group of 22 female and 13 male university students, mean age 23 years, was recruited for the cup-balancing study (Experiment 2). All participants were right-handed as assessed by the Briggs and Nebes (1975) Handedness Scale, and had normal or corrected-to-normal vision. Each student provided written, informed consent to participate and was paid for his or her involvement.

\section{Procedure}

Participants in both experiments were randomly assigned, in equal number, to a high-, low-, or neutral-power condition. Immediately before conducting either the bisection or the cup-balancing task, participants were administered a widely used power manipulation in which they wrote a narrative essay about a past event (see Galinsky et al., 2003). Participants assigned to the high-power condition wrote about a particular incident in which they had power over another individual or individuals. It was explained that this referred to a situation in which they controlled the ability of another person or persons to obtain something they wanted and/or were in a position to evaluate those individuals. In the low-power condition, participants were asked to report an incident in which someone else had power over them - that is, to report a situation in which someone had control over their ability to obtain something they wanted or was in a position to evaluate them. In the neutral condition, participants were asked to write about an event from the previous day.

A manipulation check was then performed in which all participants were asked to rate, on a scale of 1 (not at all) to 9 (very much), how much in control they felt during the incident that they had written about. Participants' ratings from the line bisection experiment were entered into a one-way ANOVA $[F(2,69)=88.8, p<.01]$ and confirmed that different levels of perceived power were reported by the low-power $(M=2.6, S D=2.4)$, high-power $(M=7.0, S D=$ $2.1)$, and neutral $(M=4.7, S D=0.5)$ conditions. The same pattern emerged when participants' ratings from the cup-balancing experiment were also entered into a one-way ANOVA $[F(2,32)=14.0$, $p<.01]$, again showing that the low-power $(M=2.1, S D=0.7)$, high-power $(M=7.4, S D=0.7)$, and neutral $(M=4.8, S D=0.5)$ participants differed in their levels of perceived power. In the cupbalancing experiment, a four-item, six-point mood questionnaire was also administered to establish whether the power manipulations induced changes in mood that might also contribute to any effects (see Keltner et al., 2003). The questionnaire asked participants to report how they felt at that current moment (sadder-happier; more discontent-more content; more tense-more relaxed; worse-better) and showed no reliable relationship between the level of perceived power and $\operatorname{mood}[F(2,30)=0.7, p=.54]$.

Experiment 1: Line bisection. Participants bisected 30 black lines that were $0.1 \mathrm{~cm}$ thick and measured $15-23 \mathrm{~cm}$ in length. Each 
line was printed individually on a separate sheet of A4 paper, the center of which was aligned with the midsagittal plane. Participants were instructed to "place a mark in the middle of the line" and were given each sheet of paper by the experimenter, who sat directly opposite. Participants were given as much time as they needed to make each bisection mark.

Experiment 2: Cup balancing. A narrow passage was created by suspending two floor-length black curtains from the ceiling. The passage was $150 \mathrm{~cm}$ long and had a width that was individually adjusted for each participant to allow $2.5 \mathrm{~cm}$ clearance on either side. The balancing task required them to "walk through the passage while balancing a full cup of water on a small tray." They were required to place their hands on opposite sides of the tray and were told that any spillages would later be measured. Participants were told to take as much time as they needed. Once they were through the passage, participants continued for a few more meters to the end of the room, where they picked up a new cup of water and began a new trial. Participants made 10 videotaped passes through the passage, during which time an assessor who was blind to power assignment recorded how many times they bumped into the left or right side of the curtain. The number of left/right collisions recorded by the assessor was later corroborated using a frame-by-frame analysis of the video footage. Spillages were not measured, although (thankfully) no one spilled an entire cup of water.

\section{RESULTS}

\section{Line Bisection}

Bisection accuracy was measured to the nearest millimeter, with a negative sign used to denote leftward errors and a positive sign to denote rightward errors. Linear contrasts showed that low-power participants produced a greater mean leftward bisection error $(-1.24 \mathrm{~cm})$ than high-power participants $(0 \mathrm{~cm})\left[t(46)=2.0, p=.03, d_{2}=5.4\right]$. There was also a nonsignificant tendency for this leftward bias to distinguish low-power participants from the neutrals $(-0.02 \mathrm{~cm})\left[t(46)=1.4, p=.09, d_{2}=4.9\right]$. No difference was found between the high-power and neutral participants $\left[t(46)=0.5, p=.63, d_{2}=0.1\right]$ (see Figure 1A). Onesample $t$ tests were also performed to determine whether the mean bisection error of each group differed significantly from zero. These revealed that whereas low-power participants erred significantly from zero $[t(23)=2.5, p>$ $.05]$, the high-power $[t(23)=0.7, p>.05]$ and neutral $[t(23)=0.6, p>.05]$ participants did not.

\section{Cup Balancing}

The number of times that each participant bumped into the left and right curtain was entered into a 3 (power: high power vs. low power vs. neutral) $\times 2$ (collision side: left vs. right) mixed-effects ANOVA, which showed the expected interaction between power and collision side $\left[F(2,32)=4.7, p=.02, \eta_{\mathrm{p}}^{2}=.3\right]$. Within-group pairwise comparisons revealed that, on average, low-power participants made a significantly greater number of right- than left-sided collisions across the 10 trials $[t(11)=3.02, p<$ $.05]$, whereas the high-power $[t(11)=0.7, p>.05]$ and neutral $[t(10)=1.9, p>.05]$ participants showed no significant left/right difference. Between-group comparisons showed that the asymmetric left/right ratio observed in the low-power group was significantly different from that seen in the high-power $[t(22)=2.25, p<.05]$ and neutral

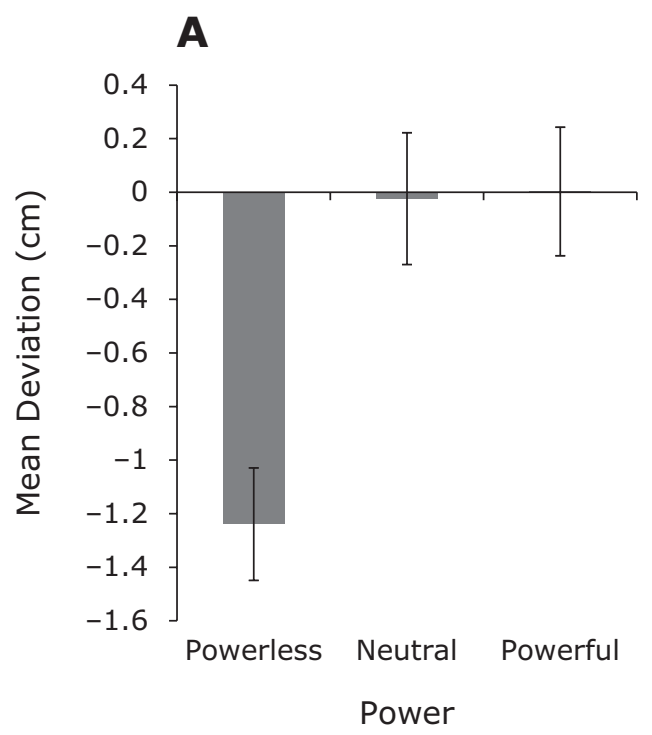

B

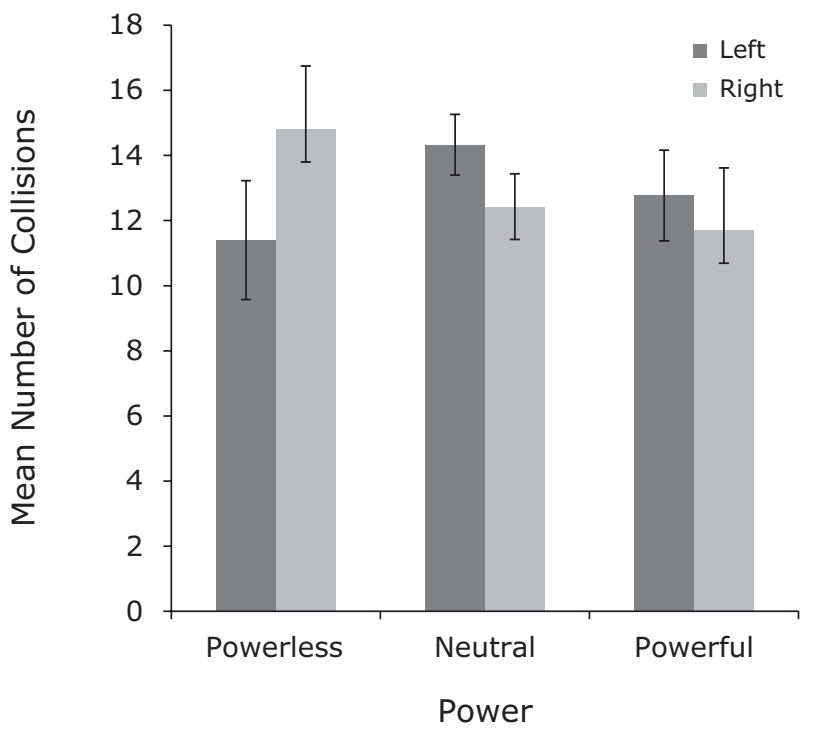

Figure 1. The effect of power (with standard error bars) on (A) line bisection accuracy and (B) the mean number of left/right collisions during the cup-balancing task.

$[t(21)=3.61, p<.05]$ groups. By contrast, the highpower and neutral groups produced comparable left/right collision ratios $[t(21)=0.46, p>.05]$. Finally, the main effects of power and collision failed to reach significance $(F \mathrm{~S}<1)$, indicating that the three groups produced the same number of total collisions, with no overall tendency for experimental participants to collide more frequently with the left or right curtain (see Figure 1B).

\section{DISCUSSION}

The data from both tasks show a leftward attentional shift in the low-power, in comparison with the high-power, 
participants. In the bisection task, those primed with low power bisected lines significantly more to the left than did their powerful counterparts and were the only group to err significantly from zero. In fact, the magnitude of bisection error (6.1\% of total line length) bordered the threshold at which the clinical condition of right-hemispatial neglect is diagnosed (Wilson et al., 1987). In the cup-balancing task, only low-power participants collided more frequently with the right side. These patterns are consistent with an increased spatial bias to the left side following preferential activation of the right hemisphere in low-power participants, showing for the first time that a change from high to low power can induce a systematic spatial bias in visuomotor performance.

From a wider perspective, our findings may appeal because they extend the idea from social psychology that social disempowerment affects the ability to set and implement goals (Guinote, 2007c; Overbeck \& Park, 2006). As mentioned, studies have shown that powerless individuals act less on opportunities and affordances provided by the environment, procrastinate before acting, and are less good at identifying opportunities to act in a goal-consistent manner. Here, we show that power affects not only if and when we choose to act, but also downstream sensorimotor processes involved in the physical control of both fine, unimanual (i.e., line bisection) and gross locomotory (i.e., walking while cup balancing) behavior.

Although the main focus of the study was to compare the effects of high and low power, the performance of the neutral participants provides some broader insight. Although no discernible differences between the high-power and neutral participants were seen, low-power participants differed from the neutrals in both tasks; only low-power participants produced a lateral bisection error that was significantly different from zero, and only the low-power participants made more right than left collisions. That said, one must be careful not to overstate the significance of these results. First, although the neutral participants in our experiment did not show pseudoneglect (i.e., err significantly from zero), control participants in other experiments have commonly done so (e.g., Braun \& Kirk, 1999; Nicholls \& Roberts, 2002). Indeed, the mean bisection error of the two groups differed from one another only marginally $(p=.09)$. Second, the failure to find a right bias in the high-power group may partly reflect the natural dominance of the right hemisphere (in right-handers) during attentional control (Kinsbourne, 1993). This hemispheric asymmetry might make it harder for high power to induce a rightward bias, even if it activated the left hemisphere as much as low power activated the right. Together, these observations encourage one to focus on the pattern of spatial bias that emerges when comparing individuals from opposite ends, as opposed to the middle part, of the social power spectrum.

In sum, we report that the relatively high-order manipulation of social power can penetrate spatial processes that are commonly considered low level. On a speculative note, if a sense of powerlessness really does preferentially activate lateralized cognitive processes in the right cerebral hemisphere, then might other types of visual process be af- fected? Face recognition and visuospatial memory are both known to rely heavily on the right hemisphere (see Hellige, 1993; Wilkinson et al., 2009), and the intriguing, albeit conjectural, question is whether tasks that engage these capacities are performed more efficiently by individuals with low, compared with high, perceived power. If true, then a curious pattern would emerge, in which powerless individuals are better than their powerful counterparts at recognizing objects, yet worse at interacting with them.

\section{AUTHOR NOTE}

This work was funded by two grants from the Economic and Social Research Council (U.K.) - one to the first and second authors (RES000-22-2716), and a separate one to the third author (PTA-026-271908). Correspondence concerning this article should be addressed to D. Wilkinson, School of Psychology, University of Kent, Canterbury, Kent CT2 7NP, England (e-mail: dtw@kent.ac.uk).

Note-Accepted by Cathleen M. Moore's editorial team.

\section{REFERENCES}

Ashby, F. G., Isen, A. M., \& Turken, A. U. (1999). A neuropsychological theory of positive affect and its influence on cognition. Psychological Review, 106, 529-550.

Boksem, M. A., Smolders, R., \& De Cremer, D. (in press). Social power and approach-related neural activity. Social, Cognitive, \& Affective Neuroscience.

Braun, J. G., \& KiRK, A. (1999). Line bisection performance of normal adults: Two subgroups with opposite biases. Neurology, 53, 527532.

Briggs, G. G., \& NebEs, R. D. (1975). Patterns of hand preference in a student population. Cortex, 11, 230-238.

DAVIDSON, R. J. (1992). Emotion and affective style: Hemispheric substrates. Psychological Science, 3, 39-43.

Fiske, S. T. (1993). Controlling other people: The impact of power on stereotyping. American Psychologist, 48, 621-628.

Galinsky, A. D., Gruenfeld, D. H., \& Magee, J. C. (2003). From power to action. Journal of Personality \& Social Psychology, 85, 453466.

Guinote, A. (2007a). Behaviour variability and the situated focus theory of power. In W. Stroebe \& M. Hewstone (Eds.), European review of social psychology (Vol. 18, pp. 256-295). New York: Wiley.

Guinote, A. (2007b). Power affects basic cognition: Increased attentional inhibition and flexibility. Journal of Experimental Social Psychology, 43, 685-697.

Guinote, A. (2007c). Power and goal pursuit. Personality \& Social Psychology Bulletin, 33, 1076-1087.

Guinote, A. (2008). Power and affordances: When the situation has more power over powerful than powerless individuals. Journal of Personality \& Social Psychology, 95, 237-252.

Guinote, A., Reese, G., \& Wilkinson, D. (in press). A powerful vision: Power affects visual search behavior. Psicología.

Hellige, J. B. (1993). Hemispheric asymmetry: What's right and what's left. Cambridge, MA: Harvard University Press.

Keltner, D., Gruenfeld, D. H., \& Anderson, C. (2003). Power, approach, and inhibition. Psychological Review, 110, 265-284.

KinsBourne, M. (1993). Orientational bias model of unilateral neglect: Evidence from attentional gradients within hemispace. In I. H. Robertson \& J. C. Marshall (Eds.), Unilateral neglect: Clinical and experimental studies (pp. 63-86). Hillsdale, NJ: Erlbaum.

LEWIN, K. (1941). Regression, retrogression, and development. University of Iowa Studies of Child Welfare, 18, 1-43.

Nicholls, M. E., Loftus, A. [M.], Mayer, K., \& Mattingley, J. B. (2007). Things that go bump in the right: The effect of unimanual activity on rightward collisions. Neuropsychologia, 37, 307-314.

Nicholls, M. E., Loftus, A. M., Orr, C. A., \& Barre, N. (2008). Rightward collisions and their association with pseudoneglect. Brain \& Cognition, 68, 166-170. 
Nicholls, M. E., \& Roberts, G. R. (2002). Can free-viewing perceptual asymmetries be explained by scanning, pre-motor or attentional biases? Cortex, 38, 113-136.

Overbeck, J. R., \& PARK, B. (2006). Powerful perceivers, powerless objects: Flexibility of powerholders' social attention. Organizational Behavior \& Human Decision Processes, 99, 227-243.

Robertson, I. H., \& Halligan, P. W. (1999). Spatial neglect: A clinical handbook for diagnosis and treatment. Hove, U.K.: Erlbaum.

Smith, P. K., Jostmann, N. B., Galinsky, A. D., \& van Dijk, W. W. (2008). Lacking power impairs executive functions. Psychological Science, 19, 441-447.

Thibaut, J. W., \& Kelley, H. H. (1959). The social psychology of groups. New York: Wiley.
Wilkinson, D., \& Halligan, P. (2004). The relevance of behavioural measures for functional-imaging studies of cognition. Nature Reviews Neuroscience, 5, 67-73.

Wilkinson, D., Ko, P., Wiriadjaja, A., Kilduff, P., McGlinchey, R., \& MilberG, W. (2009). Unilateral damage to the right cerebral hemisphere disrupts the apprehension of whole faces and their component parts. Neuropsychologia, 47, 1701-1711.

Wilson, B., Cockburn, J., \& Halligan, P. (1987). Behavioural Inattention Test. Pearson Assessment, UK.

(Manuscript received March 16, 2010; revision accepted for publication July 17, 2010.) 